\section{Intestinal Oleander Poisoning and Critical Care Management: A Rare Case Report}

\author{
Uma Hariharan ${ }^{1 *}$, Sonali Tripathi ${ }^{2}$, Pradeep Meshram ${ }^{2}$ and \\ Jaishree Doval ${ }^{2}$
}

${ }^{1}$ Department of Anesthesia and Intensive Care, Ram Manohar Lohia Hospital, New Delhi, India

${ }^{2}$ Bhagwaan Mahavir Hospital, Delhi Government Health Services, Pitampura, New Delhi, India
Cardiac glycosides cause poisoning by inhibition of $\mathrm{Na}^{+} / \mathrm{K}^{+}$ ATPase of cardiac muscles [3]. They increase the vagal tone, producing positive inotropic and negative chronotropic effect [4].

Major symptoms of oleander poisoning are cardiac and gastrointestinal in nature and appears 2-4 hours after ingestion [4,5].

We hereby report a case of accidental intestinal oleander ingestion and its successful management.

\section{Case Report}

A 26 years old male, presented to the emergency room with vomiting, disorientation, increased tone of muscles (spasticity) in lower limbs.

There was history of ingestion of yellow oleander leaves (around 6-7 leaves), about 8-10 hours prior with suicidal intent. On initial examination, the blood pressure was $110 / 64 \mathrm{mmHg}$ with irregular pulse rate of 48-52 Beats Per Minute (BPM). $\mathrm{SpO}_{2}$ was $94 \%$ on room air. He was dehydrated, drowsy and disoriented. He had aphasia along with muscle fasciculations and increased muscle tone of limbs. Pupils were bilaterally normal. All other general physical parameters were within normal limits. On systemic examination, his chest was bilaterally clear. Airway was patent with intact reflexes and adequate respiratory efforts. Cardiovascular examination revealed a slow, irregular rhythm with heart sounds soft in intensity. Per abdominal examination revealed generalized tenderness. After sending all routine investigations, gastric lavage was done with activated charcoal in the ICU (Intensive Care Unit).

The patient was given one dose $0.6 \mathrm{mg}$ of Atropine Intravenously (IV), which did not resolve his bradycardia. His heart rate further dropped to $42 \mathrm{bpm}$ from $51 \mathrm{bpm}$.

A second dose of injection Atropine $0.6 \mathrm{mg}$ intravenously was given. Heart rate increased to $45 \mathrm{bpm}$. Thereafter, IV Atropine $0.6 \mathrm{mg}$ QID (four times a day) was started. IV Phenytoin $100 \mathrm{mg}$ was given for fasciculations, which also improved the hyperrigidity. ENT (Ear, Nose and Throat) examination revealed pharyngeal edema. Steroid therapy with IV Dexamethasone $(8 \mathrm{mg}$ ) was started which improved the aphasia after 24 hours of steroid therapy.

ECG (Electrocardiogram) showed irregular rhythm with first degree AV (Atrioventricular) block. All the routine investigations were within normal limits except for serum potassium level which was $5.7 \mathrm{meq} / \mathrm{L}$ on day one of ICU admission.

All measures were taken to correct hyperkalemia (including intravenous calcium gluconate and salbutamol nebulization) and serum potassium returned to normal subsequently. Heart rate returned to normal after 3 days of admission and he was transferred from ICU on day 4 in a stable condition.

\section{Discussion}

Most of the plants, including foxglove and oleander, have been identified as containing cardiac glycosides. These include oleandrin, oleandroside, nerioside, digitoxigenin, thevetin and thevetoxin. The 
oleander seeds are highly irritant to gastrointestinal tract and the symptoms range from nausea and vomiting to cramping and bloody diarrhea $[3,6,7]$.

Oleander also causes irritation to the mucosal membranes, resulting in burning around the mouth and increased salivation. Confusion, dizziness, drowsiness, weakness, visual disturbances and mydriasis are the central nervous system manifestations of toxicity $[4,8]$. The most serious side effects of oleander poisoning are cardiac abnormalities, including various ventricular dysrhythmias, bradycardia and heart blocks $[4,9]$.

ECG often reveals an increased PR interval, a decreased QT interval and $\mathrm{T}$ wave flattening or inversion. It is thought that these clinical manifestations are the result of both increased vagal tone and direct cardiac glycoside toxicity $[4,6]$.

The treatment of oleander poisoning is empirically based on treatment of digitalis glycoside toxicity and consists of supporting the patient haemodynamically. This includes administration of injection Atropine for severe bradycardia and using injection Phenytoin or injection Lignocaine hydrochloride to control dysrhythmias. In severe intractable cases, placing a temporary pacemaker, DC.

Shock and administration of digoxin specific Fab antibody fragments (Digibind) should be considered $[4,8]$. Specific Fab antibodies were not used in this patient as it was not a severe, intractable poisoning case and he responded to standard supportive care. Other treatment methods are aimed at removing the toxic substance from the stomach by emesis. Special precaution should be given to a patient with bradycardia before emesis is induced because of the possibility of a vagal stimulation and worsening of bradycardia [4]. Although, our patient was brought after 8-10 hours of ingestion of the toxin, but activated charcoal was still used to remove any residual toxin from the stomach. Cardiac steroid levels were not measured in our patient as facilities for its measurement were not available in our hospital.

\section{Conclusion}

Oleander poisoning can be fatal with relatively small amounts ingested. The calculated lethal oleander leaf dose was found to be approximately $4 \mathrm{gm}$ in previous studies [6]. Some of the cardiac glycosides and hydro-methalonic extracts of oleander are also known for their pharmacologic properties, as this plant is extensively used for treatment of diverse ailments in complimentary and alternative medicine. Hence, therapeutic activity or poisonous nature of oleander depends upon the dose or concentration. Intensivists and emergency physicians should understand the potential lethal properties of oleander and its availability throughout the world, especially in India and Sri Lanka (Asian continent). Prompt recognition of the toxic symptoms and its expeditious management gives a successful outcome.

\section{References}

1. Frohne DP, Fander HJ (1984) A Colour Atlas of Poisonous Plants: A Handbook for Pharmacists, Doctors, Toxicologists, and Biologists. Wolfe Publishing Itd., London, UK.

2. Eddleston M, Ariaratnam CA, Meyer WP, Perera G, Kularatne AM, et al (1999) Epidemic of self-poisoning with seeds of the yellow oleander tree (Thevetia Peruviana) in northern Sri Lanka. Trop Med Int Health 4: 266-273.

3. Galey FD, Holstege DM, Plumlee KH, Tor E, Johnson B, et al. (1996) Diagnosis of oleander poisoning in livestock. J Vet Diagn Invest 8: 358-364.

4. Khan I, Kant C, Sanwaria A, Meena L (2010) Acute cardiac toxicity of nerium oleander/indicum poisoning (kaner) poisoning. Heart Views 11: 115-116.

5. Al B, Yarbil P, Dogan M, KabulS, YIIdırım C (2010) A case of non-fatal oleander poisoning. BMJ Case Rep.

6. Osterloh J, Herold S, Pond S (1982) Oleander interference in the digoxin radioimmunoassay in a fatal ingestion. JAMA 247: 1596-1597.

7. Everist SL (1981) Apocynaceae In: Poisonous plants of Australia. Angus and Robertson, London. Pg no 2: 77-89.

8. Shumaik GM, Wu AW, Ping AC (1988) Oleander poisoning: Treatment with digoxin specific Fab antibody fragments. Ann Emerg Med 17: 732-735.

9. Ansford AJ, Morris H (1981) Fatal oleander poisoning. Med J Aust 1: 360361. 